\title{
Intraosseous Rosai-Dorfman disease diagnosed by touch imprint cytology evaluation: A case series
}

\author{
Avani A. Pendse, MBBS, PhD 1,2 (D) | Sara E. Wobker, MD, MPH² | \\ Kevin G. Greene, MD ${ }^{2}$ | Scott V. Smith, MD2 \\ Robert J. Esther, MD3 | Leslie G. Dodd, MD²
}

${ }^{1}$ Department of Pathology, Duke University Medical Center, Durham, North Carolina

${ }^{2}$ Department of Pathology and Laboratory Medicine, University of North Carolina at Chapel Hill, Chapel Hill, North Carolina

${ }^{3}$ Department of Orthopedics, University of North Carolina at Chapel Hill, Chapel Hill, North Carolina

\section{Correspondence}

Avani Pendse, MBBS PhD, Department of Pathology, Duke University Medical Center, Box 3712, Durham, NC 27710.

Email: avani.pendse@duke.edu

\begin{abstract}
Sinus histiocytosis with massive lymphadenopathy, also known as Rosai-Dorfman disease (RDD) is a rare benign disorder that primarily affects the lymph nodes. Localized lymphadenopathy is the most common clinical manifestation of this disorder. However, RDD has been described in several extra-nodal sites including the head and neck region, soft tissue, skin, upper respiratory tract, gastro-intestinal tract and central nervous system (CNS). Involvement of the bone is considered very rare, occurring in less than $10 \%$ patients. RDD is one of the histiocytoses and the differential diagnosis includes entities such as Langerhans cell histiocytosis and Erdheim-Chester disease. In the rare intraosseous variant, the clinical and radiologic differential diagnosis is broader and includes neoplasms such as osteosarcoma and Ewing sarcoma. In this report, we describe three cases of extra-nodal, intraosseous RDD where touch imprint cytology played a crucial role in diagnosis. Two of the cases initially presented with involvement of the head and neck region and later developed intraosseous disease; while the third patient presented with primary bone involvement. The diagnosis was established by core biopsy with touch imprints of the bone lesions. The cytologic samples showed numerous histiocytes, often with neutrophils within their cytoplasm (emperipolesis) in addition to lymphocytes and plasma cells. The diagnosis of RDD was confirmed with appropriate immunohistochemical stains. Our account of these three cases of intraosseous Rosai-Dorfman disease highlights the role of cytology in the diagnosis of this rare entity.

\section{KEYWORDS}

cytology, intra-osseous, Rosai-Dorfman disease, touch imprint
\end{abstract}

\section{1 | INTRODUCTION}

Sinus histiocytosis with massive lymphadenopathy, also known as Rosai-Dorfman disease (RDD) was comprehensively described by Drs. Juan Rosai and Ronald Dorfman following an initial description by Destombes in $1965 .{ }^{1-3}$ This disease often presents as a pseudolymphomatous disorder characterized clinically as a benign entity with a protracted clinical course and ultimate resolution in most cases. Localized lymphadenopathy is the usual clinical manifestation, and in some patients, a short nonspecific febrile episode may precede lymphadenopathy. Although initially reported in children and young adults, RDD patients show a wide age range, with a mean of 20.6 years. ${ }^{4}$ The etiology of RDD largely remains unknown. Rare cases reported in family members suggest a genetic basis. ${ }^{5}$ An infectious etiology has also been hypothesized although a specific causative microorganism has not been implicated to date.

The affected lymph nodes of RDD are enlarged with distorted architecture due to dilatation of sinuses with a mixed population of histiocytes, lymphocytes, and plasma cells. The pathologic hallmark of RDD is the characteristic engulfment of lymphocytes and other cells by histiocytes that have increased phagocytic activity. ${ }^{6}$ While bilateral cervical lymphadenopathy is the classic presentation of disease, involvement of other lymph nodes and extra-nodal sites such as head and neck, soft tissue, skin, upper respiratory tract, gastro-intestinal tract, and CNS is common. ${ }^{4}$ Involvement of the bone is somewhat rare, seen in $<10 \%$ of patients and primary intraosseous disease has only been 
reported in a small number of patients. ${ }^{7}$ In the following account, we report three cases of extra-nodal, intraosseous Rosai-Dorfman disease, where the diagnosis of RDD was established on touch imprint cytology and core biopsies of the bone lesions.

\section{2 | CASE REPORTS}

\section{$2.1 \mid$ Case 1}

A 17-year-old African American female initially presented at age nine with a 2-year history of nasal congestion. Magnetic resonance imaging (MRI) revealed a benign-appearing midline enhancing mass in the nasal septum. Endoscopic excisional biopsy of the mass revealed severe chronic inflammation and sclerosing fibrosis. No evidence of granuloma, lymphoma, or malignancy was identified and AFB, Gomori Methenamine Silver and PAS-D stains were negative for microorganisms. The patient's symptoms partially resolved following the biopsy and further laboratory evaluation was not suggestive of an underlying connective tissue disorder.

Approximately 2.5-years after her nasal biopsy, she developed left knee pain, aggravated with weight bearing and activity. X-ray of this knee revealed a metaphyseal based, partially lytic lesion of the left distal femur (Figure 1A). MRI showed a $>3 \mathrm{~cm}$ multilobulated cystic mass within the distal left femoral metaphysis and epiphysis. Clinical and radiologic differential diagnoses included osteomyelitis, primary bone tumor including Ewing sarcoma, giant cell tumor and Langerhans cell histiocytosis. A computerized tomography (CT) - guided biopsy of the lesion was performed and Diff-Quik stained touch imprints revealed multiple histiocytes with intact lymphocytes, plasma cells and occasional neutrophils within their cytoplasm (emperipolesis) (Figure 1B). These histiocytes were S-100 and CD68 positive on immunohistochemistry performed on the core biopsy specimen (Figure 1D). Based on these results, the left distal femur lesion was diagnosed as "consistent with extranodal Rosai-Dorfman disease". The earlier nasal biopsy was reviewed after the bone diagnosis was rendered and showed rare histiocytes with numerous plasma cells and lymphocytes and was reevaluated as RDD (Figure 1C). The patient was treated with bone curettage with grafting and continues care at our institution.

\section{2 | Case 2}

A 38-year-old African American female presented with a 5-year history of chronic rhinosinusitis, and was status post endoscopic sinus surgery with bilateral inferior turbinate submucosal resection. The patient continued to be symptomatic post-surgery and was worked up for vasculitis, sarcoidosis, and other autoimmune causes. Maxillo-facial CT scan
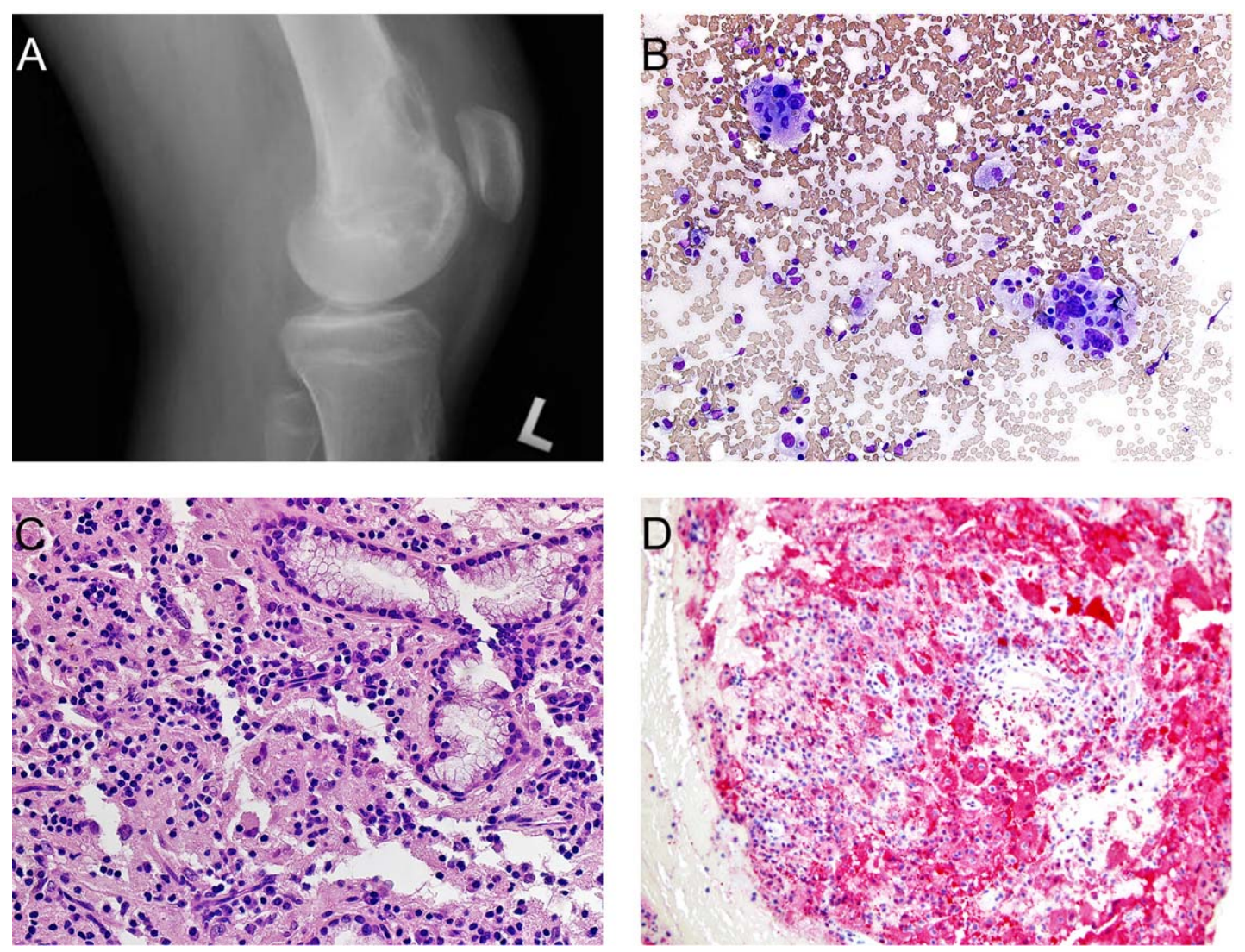

FIGURE 1 A, X-ray of the left knee showing a metaphyseal based, partially lytic lesion of the left distal femur (case 1); B, diff-Quik stained touch imprints showing multiple histiocytes with pathognomonic emperipolesis or intact lymphocytes, plasma cells and occasional neutrophils within their cytoplasm (case 1); C, histopathologic evaluation of the nasal biopsy showing scattered histiocytes with numerous plasma cells and lymphocytes (case 1); D, histiocytes showing positive S100 immunostaining performed on the core biopsy specimen (case 1) [Color figure can be viewed at wileyonlinelibrary.com] 
showed post-surgical changes, exuberant inferior turbinate hypertrophy and increased soft tissue thickening along the medial aspect of the ethmoid cavities and superior nasal cavities suggestive of polyposis. The clinical differential diagnoses included chronic rhinosinusitis, granulomatosis with polyangiitis, allergic rhinitis, nasal obstruction, and inferior turbinate hypertrophy.

She underwent a revision surgery and the histopathologic evaluation of the bilateral sinus contents revealed severe histiocytosis and mixed chronic inflammatory cells including plasma cells and lymphocytes with rare eosinophils and neutrophils. AFB, Gomori Methenamine Silver and Warthin-Starry stains were negative for microorganisms. Shortly thereafter, she complained of right shoulder pain and a lytic lesion of the right proximal humerus was identified (Figure 2A). Positron-emission-tomography (PET/CT) scan revealed diffuse bone marrow hypermetabolism with opacification of maxillary sinuses and bilateral, enlarged, intensely hypermetabolic cervical lymph nodes. In an effort to further characterize the disease process, a CT-guided bone biopsy of the humeral lesion was performed. Touch imprints of the biopsy revealed numerous histiocytes with emperipolesis in a background of reactive lymphocytes and plasma cells (Figure 2B). In the context of clinical and radiologic findings, cytologic evaluation definitively established the diagnosis of extranodal/intra-osseous RDD. 1year post diagnosis she had not experienced any disease progression.

\section{3 | Case 3}

A 17-year-old African American male presented with increasing left leg and knee pain for about 3-years. X-rays showed multifocal aggressive bone lesions in bilateral femurs, left greater than right. Lytic lesions also involved both distal and proximal tibia and proximal left humerus. Subsequent MRI showed expansile bone lesions centered in the medullary canals of the left and right femurs with cortical erosion and breakthrough with soft tissue expansion in the distal femur. A destructive enhancing lesion was also identified in the proximal left humerus. A $\mathrm{PET} / \mathrm{CT}$ showed multiple FDG-avid bone lesions. Additionally, peri-aortic/retroperitoneal fibrosis was identified on the CT scan. Notably, there was no evidence of lymphadenopathy on clinical examination or radiology.

The clinical and radiologic differential diagnosis was broad and included Ewing sarcoma, osteosarcoma, osteomyelitis, Langerhans cell histiocytosis and lymphoma. The possibility of Erdheim-Chester disease (ECD) was raised in the presence of radiographic findings of peri-aortic/retroperitoneal fibrosis. The patient underwent two CT-guided fine needle aspirations and core needle biopsies of the left femur. The significant finding on touch imprint was a prominent histiocytosis with focal collections of large cells with striking emperipolesis (Figure $2 \mathrm{C}$ and 2D). While ECD was considered in the differential diagnosis, the morphology and immunophenotype of the large cells (S-100, Fascin,
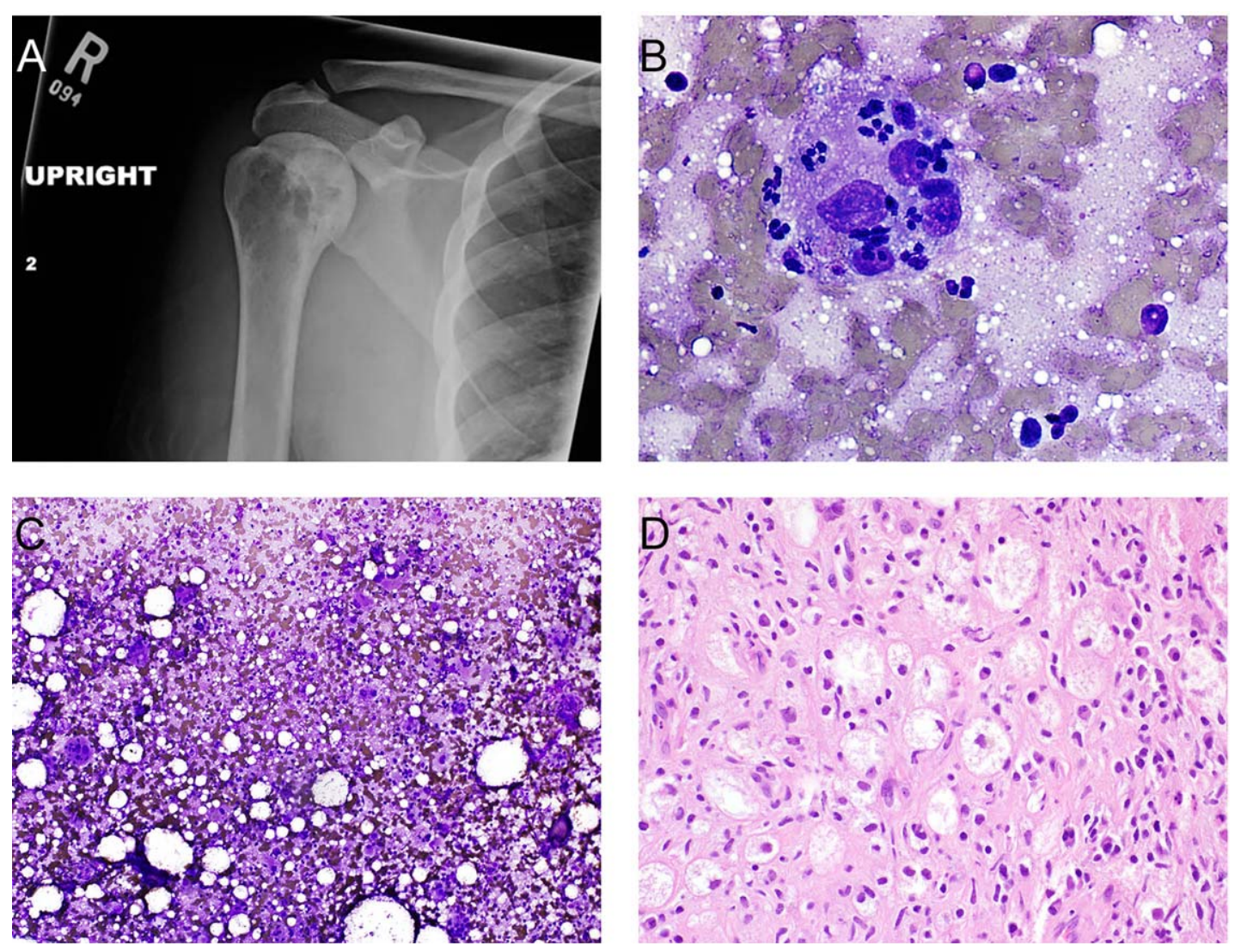

FIGURE 2 A, X-ray of the right shoulder showing a lytic lesion of the proximal humerus (case 2); B, additional depiction of emperipolesis in Diff-Quik stained touch imprints (case 2); C, cellular fine needle aspirate with numerous histiocytes (case 3); D, H\&E correlate of aspirate in panel $\mathrm{C}$ with numerous histiocytes with emperipolesis (case 3) [Color figure can be viewed at wileyonlinelibrary.com] 
CD163, CD68 positive; Factor XIII negative) were most suggestive of RDD.

\section{3 | DISCUSSION}

Sinus histiocytosis with massive lymphadenopathy or RDD was originally described as a disease of the lymph nodes. After the initial description of classic RDD involving the nodes, it became clear that RDD was a multi-organ process in a subset of patients. Bone involvement, as either primary disease or in addition to RDD of lymph nodes or soft tissues, is still considered very rare. ${ }^{4,7,8}$ Similar to our three examples, affected patients typically present with pain or swelling of the affected bone, often accompanied by symptomatic involvement of other sites and lymphadenoapathy. Rare cases have been reported to be asymptomatic and incidentally discovered on imaging studies. ${ }^{9}$ Imaging of bones involved with RDD often reveals lytic lesions of the medullary cavity, but a specific radiologic diagnosis is seldom given. ${ }^{10,11}$ Additionally, RDD involvement can show increased metabolic activity on fluorodeoxy-glucose positron-emission-tomography (FDG-PET) scans, making it difficult to distinguish from malignancy.

The cytologic and histologic features of RDD include a diffuse inflammatory infiltrate comprised of plasma cells, lymphocytes and numerous histiocytes. The latter are often large with abundant cytoplasm containing engulfed neutrophils, lymphocytes, plasma cells and erythrocytes. ${ }^{6}$ Nuclei are usually large with a distinct, central nucleolus. The histiocytes often contain intracytoplasmic vacuoles and show emperipolesis, characterized by engulfed inflammatory cells. Histiocytes in RDD disease are usually positive for CD68 and S-100 and are negative (or occasionally weakly positive) for CD1a. ${ }^{12-14}$ This immunohistochemical staining pattern is not only used to support the diagnosis but also to rule out specific entities such as Langerhans cell histiocytosis (LCH) from the differential diagnosis. Other differential diagnoses such as Erdheim-Chester disease, hemophagocytic syndrome, osteomyelitis, primary bone tumors, primary bone lymphoma and metastatic disease can also be reliably excluded on cytology specimens in the context of appropriate clinical history and use of ancillary tests.

Since the initial recognition and description of RDD almost 45 years ago, there have been several reports of the cytologic findings. ${ }^{8,15-30}$ Among these are cases of RDD in unusual locations including the breast, ${ }^{19}$ orbit, ${ }^{23,28}$ thyroid, ${ }^{29}$ gastrointestinal tract, ${ }^{30}$ and primary involvement of the bone. ${ }^{8,22,26,27}$ Almost every documented cytologic report describes what appears to be the sine quo non for diagnosis - emperipolesis, also termed lymphophagocytosis. While most authors have suggested that FNA may "contribute" or may represent a "useful diagnostic procedure", the diagnosis was eventually confirmed by a surgical biopsy. Alternatively, initial biopsies have been reported to be diagnostically challenging in a subset of RDD patients. ${ }^{7}$ As occurred in our cases, the lesional histiocytes may be obscured due to hypercellularity and mixed chronic inflammation in fixed tissue sections. Touch imprints can be effectively used in diagnosing RDD where the characteristic emeripoietic histiocytes in background of reactive lymphocytes and plasma cells can be readily demonstrated. Based on our experience, in agreement with a limited number of previously published accounts, FNA with core biopsy of RDD is diagnostic in itself and excisional biopsy may be avoided completely in a majority of cases. $^{20,22,24,25}$

\section{ORCID}

Avani A. Pendse MBBS, PhD (ID http://orcid.org/0000-0002-9243-8047

\section{REFERENCES}

[1] Destombes P. Adenitis with lipid excess, in children or young adults, seen in the Antilles and in Mali (4 cases) [in French]. Bull Soc Pathol Exot Filiales 1965;58:1169-1175.

[2] Rosai J, Dorfman RF. Sinus histiocytosis with massive lymphadenopathy. A newly recognized benign clinicopathological entity. Arch Pathol 1969;87:63-70.

[3] Rosai J, Dorfman RF. Sinus histiocytosis with massive lymphadenopathy: a pseudolymphomatous benign disorder. Analysis of 34 cases. Cancer 1972; 30:1174-1188.

[4] Foucar E, Rosai J, Dorfman R. Sinus histiocytosis with massive lymphadenopathy (Rosai- Dorfman disease): review of the entity. Semin Diagn Pathol 1990;7:19-73.

[5] Kismet E, Koseoglu V, Atay AA, Deveci S, Demirkaya E, Tuncer K. Sinus histiocytosis with massive lymphadenopathy in three brothers. Pediatr Int 2005;47:473-476.

[6] loachim HL, Medeiros LJ. Sinus histiocytosis with massive lymphadenopathy. In: loachim HL, Medeiros LJ, ed. loachim's Lymph Node Pathology. Lippincott Williams \& Wilkins; 2009. P193198.

[7] Demicco EG, Rosenberg AE, Bjornsson J, Rybak LD, Unni K, Nielsen GP. Primary Rosai-Dorfman disease of bone. A clinicopathologic study of 15 cases. Am J Surg Pathol 2010;34:1324-1333.

[8] Jing X, McHugh JB, Pu RT. Fine-needle aspiration cytology of RosaiDorfman disease of bone. Diagn Cytopathol 2008;36:516-518.

[9] Nawroz IM, Wilson-Storey D. Sinus histiocytosis with massive lymphadenopathy (Rosai-Dorfman disease). Histopathol 1989;14:91-99.

[10] McAlister WH, Herman T, Dehner LP. Sinus histiocytosis with massive lymphadenopathy (Rosai- Dorfman disease). Pediatr Radiol 1990;20:425-432

[11] Raslan OA, Schellingerhout D, Fuller GN, Ketonen LM. Rosai- Dorfman disease in neuroradiology: imaging findings in a series of 10 patients. AJR 2011;196:W187-W193.

[12] Gattuso P, Reddy BV, David O, Spitz JD, Haber HM. Sinus histiocytosis with massive lymphadenopathy (Rosai- Dorfman disease). In: Haber HM, ed. Differential Diagnosis in Surgical Pathology. 2nd ed. Philadelphia: Saunders; 2010. P763765.

[13] Lopez P, Estes ML. Immunohistochemical characterization of the histiocytes in sinus histiocytosis with massive lymphadenopathy: analysis of an extranodal case. Hum Pathol 1989;20:711-715.

[14] Eisen RN, Buckley PJ, Rosai J. Immunohistochemical characterization of sinus histiocytosis with massive lymphadenopathy (RosaiDorfman disease). Semin Diagn Pathol 1990;7:74-82.

[15] Layfield LJ. Fine needle aspiration cytologic findings in a case of sinus histiocytosis with massive lymphadenopathy (Rosai-Dorfman syndrome). Acta Cytol 1990;34:767-770.

[16] Trautman BC, Stanley MW, Goding GS, Rosai J. Sinus histiocytosis with massive lymphadenopathy (Rosai-Dorfman disease): diagnosis by fine-needle aspiration. Diagn Cytopathol 1991;7:513-516. 
[17] Alvarez Alegret R, Martinez Tello A, Ramirez T, et al. Sinus histiocytosis with massive lymphadenopathy (Rosai-Dorfman disease): diagnosis with fine-needle aspiration in a case with nodal and nasal involvement. Diagn Cytopathol 1995;13:333-335.

[18] Deshpande V, Verma K. Fine needle aspiration (FNA) cytology of Rosai-Dorfman disease. Cytopathology 1998;9:329-335.

[19] Hummel P, Waisman J, Chhieng D, et al. Fine-needle aspiration cytology of Rosai-Dorfman disease of the breast: A case report. Diagn Cytopathol 1999;21:287-291.

[20] Deshpande AH, Nayak S, Munshi MM. Cytology of sinus histiocytosis with massive lymphadenopathy (Rosai-Dorfamn disease). Digan Cytopathol 2000; 22:181-185.

[21] Das DK, Gulati A, Bhatt NC, Sethi GR. Sinus histiocytosis with massive lymphadenopathy (Rosai-Dorfman disease): report of two cases with fine-needle aspiration cytology. Diagn Cytopathol 2001;24:42-45.

[22] Goel MM, Agarwal PK, Agarwal S. Primary Rosai-Dorfman disease of bone without lymphadenopathy diagnosed by fine needle aspiration cytology. A case report. Acta Cytol 2003;47:1119-1122.

[23] Malur RR, Bannur HB, Kodkany SB. Orbital Rosai-Dorfman disease report of a case with fine needle aspiration cytology and histopathology. Acta Cytol 2007;51:581-582.

[24] Kumar B, Karki S, Paudyal P. Diagnosis of sinus histiocytosis with massive lymphadenopathy (Rosai-Dorfman disease) by fine needle aspiration cytology. Diagn Cytopathol 2008;36:691-695.

[25] Kushwaha R, Ahluwalia C, Sipayya V. Diagnosis of sinus histiocytosis with massive lymphadenopathy (Rosai-Dorfman Disease) by fine needle aspiration cytology. J Cytol 2009;26:83-85.
[26] Li S, Yan Z, Jhala N, Jhala D. Fine needle aspiration diagnosis of Rosai-Dorfman disease in an osteolytic lesion of bone. Cytojournal 2010; 27:12. https://doi.org/10.4103/1742-64 13.65058.

[27] Schein C, Kluskens L, Gattuso P. Fine-needle aspiration of primary Rosai-Dorfman disease of the bone without peripheral lymphadenopathy: a challenging diagnosis. Diagn Cytoapthol 2013;41:230231.

[28] Majumdar K, Tyagi I, Saran RK, Kumar S, Gondal R. Multicentric extranodal Rosai-Dorfman disease-a cytological diagnosis, with histological corroboration. Acta Cytol 2012;56:214-218.

[29] Vujhini SK, Kolte SS, Satarkar RN, Srikanth S. Fine needle aspiration diagnosis of Rosai-Dorfman disease involving thyroid. J Cytol 2012; 29:83-85.

[30] Ioannidis I, Manlakis C, Laurini JA, et al. Rectal extranodal RosaiDorfman disease diagnosed by EUS-FNA: a case report and review of the literature. Diagn Cytopathol 2015;43:40-44.

How to cite this article: Pendse AA, Wobker SE, Greene KG, Smith SV, Esther RJ, Dodd LG. Intraosseous Rosai-Dorfman disease diagnosed by touch imprint cytology evaluation: A case series. Diagnostic Cytopathology. 2018;46:83-87. https://doi. org/10.1002/dc.23802 